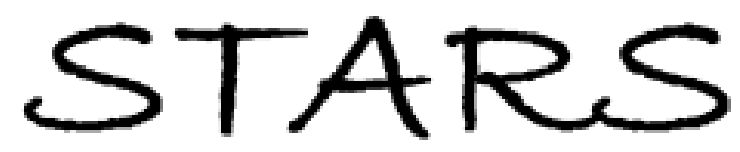

University of Central Florida

STARS

$1-1-2014$

\title{
Subpicosecond adiabatic rapid passage on a single semiconductor quantum dot: Phonon-mediated dephasing in the strong-driving regime
}

Reuble Mathew

Eric Dilcher

Angela Gamouras

Ajan Ramachandran

Hong Yi Shi Yang

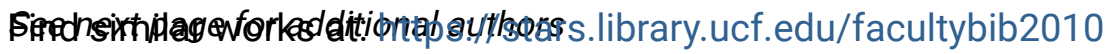

University of Central Florida Libraries http://library.ucf.edu

This Article is brought to you for free and open access by the Faculty Bibliography at STARS. It has been accepted for inclusion in Faculty Bibliography 2010 s by an authorized administrator of STARS. For more information, please contact STARS@ucf.edu.

\section{Recommended Citation}

Mathew, Reuble; Dilcher, Eric; Gamouras, Angela; Ramachandran, Ajan; Yang, Hong Yi Shi; Freisem, Sabine; Deppe, Dennis; and Hall, Kimberley C., "Subpicosecond adiabatic rapid passage on a single semiconductor quantum dot: Phonon-mediated dephasing in the strong-driving regime" (2014). Faculty Bibliography 2010s. 5805.

https://stars.library.ucf.edu/facultybib2010/5805

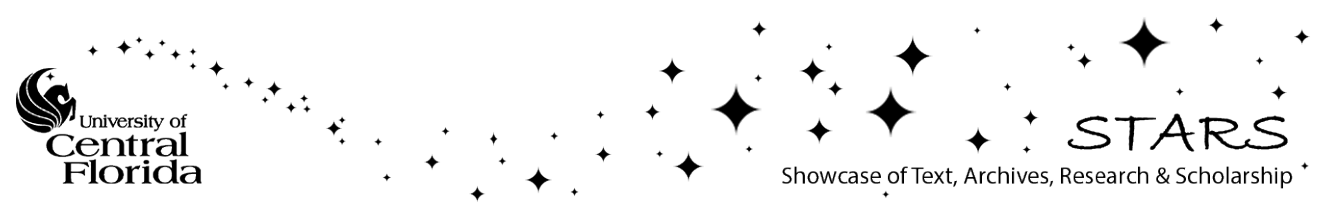




\section{Authors}

Reuble Mathew, Eric Dilcher, Angela Gamouras, Ajan Ramachandran, Hong Yi Shi Yang, Sabine Freisem, Dennis Deppe, and Kimberley C. Hall 


\title{
Subpicosecond adiabatic rapid passage on a single semiconductor quantum dot: Phonon-mediated dephasing in the strong-driving regime
}

\author{
Reuble Mathew, ${ }^{1}$ Eric Dilcher, ${ }^{1}$ Angela Gamouras, ${ }^{1}$ Ajan Ramachandran, ${ }^{1}$ Hong Yi Shi Yang, ${ }^{1}$ \\ Sabine Freisem, ${ }^{2}$ Dennis Deppe, ${ }^{2}$ and Kimberley C. Hall ${ }^{1}$ \\ ${ }^{1}$ Department of Physics and Atmospheric Science, Dalhousie University, Halifax, Nova Scotia B3H4R2, Canada \\ ${ }^{2}$ The College of Optics and Photonics, University of Central Florida, Orlando, Florida 32816-2700, USA \\ (Received 6 February 2014; revised manuscript received 23 June 2014; published 25 July 2014)
}

\begin{abstract}
We demonstrate adiabatic rapid passage on a subpicosecond time scale in a single semiconductor quantum dot, enabling the exploration of a regime of strong (and rapidly varying) Rabi energies for optical control of excitons. An observed dependence of the exciton inversion efficiency on the sign of the pulse chirp demonstrates the dominance of phonon-mediated dephasing, which is suppressed for positive chirp at low temperature. Our findings will support the realization of dynamical decoupling strategies and suggest that multiphonon emission and/or non-Markovian effects should be taken into account.
\end{abstract}

DOI: 10.1103/PhysRevB.90.035316

PACS number(s): 78.67.Hc, 71.38.-k, 78.55.Cr

\section{INTRODUCTION}

Semiconductor quantum dots (QDs) are attractive for a variety of applications in quantum information science as they offer atomlike properties in a solid-state environment, coupled with the ease and efficiency of quantum state manipulation using coherent optical pulses [1-6]. Adiabatic rapid passage (ARP) provides a particularly effective strategy for achieving quantum state inversion in these systems because, unlike optical control via Rabi oscillations [1-3], ARP is insensitive to variations in the QD parameters (dipole moment, transition energy) that are inherent in the natural size variations present in such systems. The robustness of quantum state inversion via ARP is due to the use of frequency-swept optical pulses, which result in a transfer of the system through an anticrossing for which the final state is uniquely identified with the exciton after the laser pulse is over [7-12]. Building upon demonstrations in atomic systems [7,8], ARP was recently achieved experimentally in single semiconductor quantum dots $[13,14]$, paving the way for application to efficient single and entangled photon sources $[15,16]$, quantum gates [11,17-20], all-optical switches [9,12], and the realization of a Bose-Einstein condensate in a QD ensemble [21].

Despite this promise, quantum state control in QDs is impeded by coupling to phonons, representing an intrinsic source of decoherence within the solid-state environment. The need to both elucidate and mitigate phonon-mediated decoherence processes in optical control experiments on semiconductor QDs has stimulated a comprehensive research effort in recent years [9-11,22-33]. For ARP, the impact of phonons has recently been predicted to depend on the magnitude of the peak Rabi energy during the control pulse as well as the direction of the sweep of the instantaneous pulse frequency (i.e., the sign of the pulse chirp) $[10,25,26]$. The former prediction is consistent with the observation of a drop in the final exciton occupation with increasing pulse area above the threshold for ARP [13,14]. A dependence on pulse chirp, however, provides a more transparent means of distinguishing phonon-induced dephasing from other sources of decoherence. In particular, in previous studies of damping of Rabi oscillations, coupling of the optically excited exciton with phonons $[22,23]$ or unintended carriers excited into the wetting layer [34,35] have been identified as potential sources of decoherence. For control via ARP, only the phonon-related process depends on the sign of the pulse chirp, allowing for the experimental isolation of phonon-related decoherence effects in optical control.

In the experiments reported here, we show that phonons represent the primary source of dephasing in optical control experiments on excitons in single semiconductor QDs by revealing a dependence of the exciton inversion efficiency on the sign of the control pulse chirp: For positively chirped pulses, the system traverses the lower-energy adiabatic branch, resulting in greater exciton inversion, consistent with suppression of phonon emission processes. Our experiments also represent a demonstration of ARP in an experimental regime of large Rabi energy through the use of shorter optical pulses (representing a factor of $\gtrsim 20$ reduction in comparison to previous work $[13,14])$. In addition to enabling a larger number of quantum operations within the decoherence time, the ultrafast control pulses used in the experiments reported here lay the groundwork for realizing decoherence protection via dynamical decoupling [36-39]. Comparing our experimental results with a theoretical model including phonon-mediated decoherence [22-24] suggests the importance of multiphonon emission processes and non-Markovian effects, with implications for the theoretical design of optimal control pulses that minimize decoherence. The use of short pulses also reduces the magnitude of the spectral chirp required for ARP, facilitates the accurate application of chirp via readily available commercial pulse-shaping systems, and provides a means to further optimize the quantum state evolution and to realize parallel quantum gates on multiple quantum dots through the use of general pulse-shaping techniques [20,27,40-42].

\section{EXPERIMENTAL DETAILS}

The sample under study is an InAs/GaAs quantum-dot structure grown using molecular beam epitaxy. We spectrally isolate single QDs from the ensemble using a metallic mask with a $0.4-\mu \mathrm{m}$ aperture. The sample is held on a nanopositioning stage in a continuous-flow cryostat at $10 \mathrm{~K}$. The optical source is a 76-MHz optical parametric oscillator (OPO) 
(a)

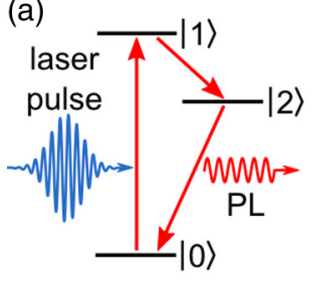

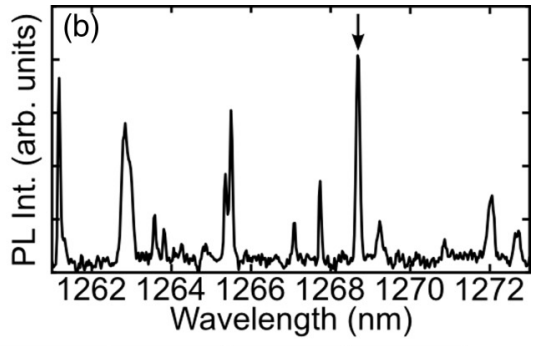
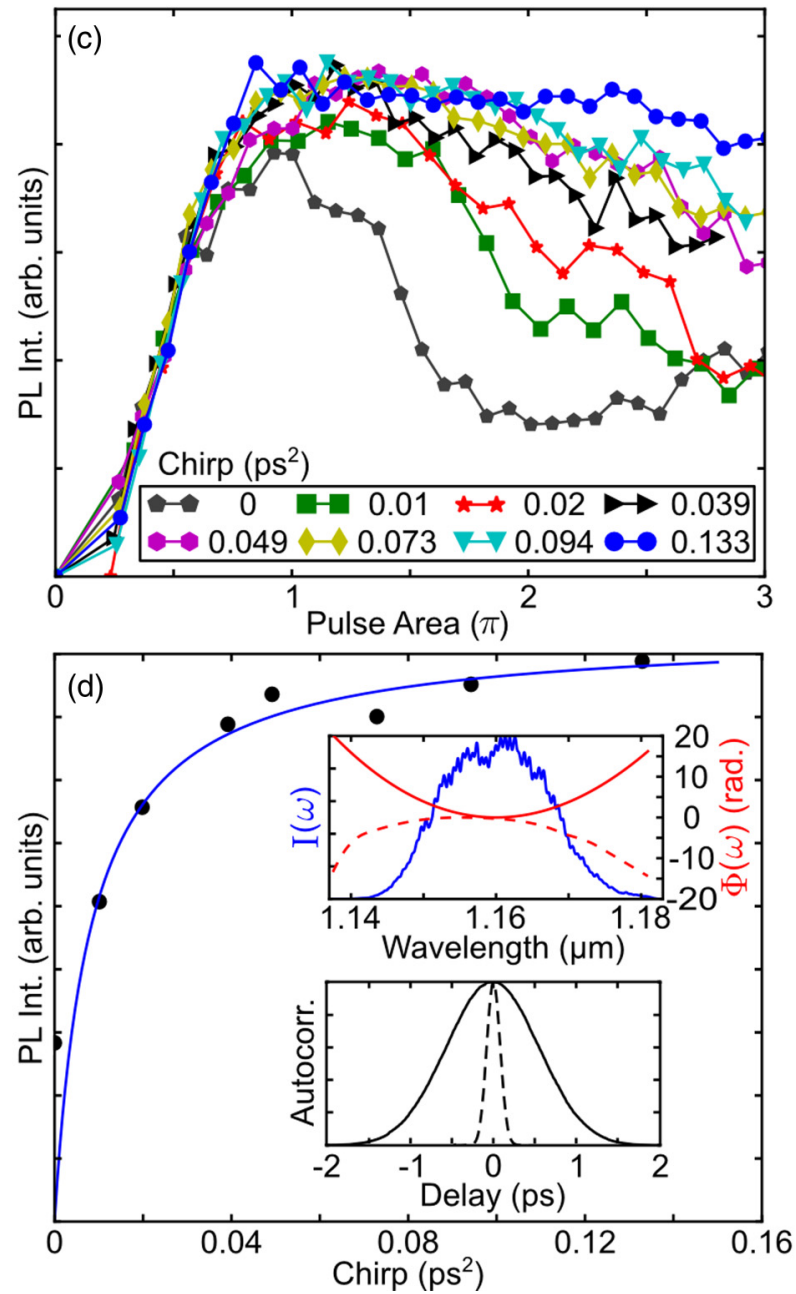

FIG. 1. (Color online) (a) Quantum state control and readout scheme with the ground state $(|0\rangle), p$-shell exciton $(|1\rangle)$, and $s$-shell exciton (|2〉). (b) Spectrally resolved PL from the $s$ shell. The arrow marks the $s$-shell emission for the QD studied here. (c) PL intensity as a function of pulse area for increasing positive chirp. (d) PL intensity at $\Theta=2 \pi$ as a function of the magnitude of the chirp (black circles) and a fit to a saturation function $\left[I=I_{0} \phi^{\prime \prime} /\left(\phi^{\prime \prime}+\phi_{\mathrm{sat}}^{\prime \prime}\right)\right]$, with $\phi_{\mathrm{sat}}^{\prime \prime}=$ $0.0083 \mathrm{ps}^{2}$ (blue curve). Inset: (top) Laser pulse spectrum (solid blue curve), dispersion compensation mask (dashed red curve), and the chirp mask applied to the dispersion-compensated pulse for $\phi^{\prime \prime}=$ $0.039 \mathrm{ps}^{2}$ (solid red curve). (bottom) Measured pulse autocorrelation for the transform-limited pulse with $\tau_{0}=120 \mathrm{fs}$ (dashed curve) and a chirped 910-fs pulse with $\phi^{\prime \prime}=0.039 \mathrm{ps}^{2}$ (solid curve).

generating $1.16-\mu \mathrm{m}$ pulses with a dispersion-compensated pulse width of $120 \mathrm{fs}$. Dispersion compensation [43] and the introduction of chirp is achieved by passing the pulses through a $4 f$ pulse shaper, with a dual-mask, 128-pixel spatial light modulator at the Fourier plane. The laser pulses, resonant with the crystal ground state $(|0\rangle)$ to $p$-shell exciton $(|1\rangle)$ transition in the QD [see Fig. 1(a)], are focused onto the sample using a high-resolution objective lens (numerical aperture of 0.7 ). Relaxation to the $s$-shell exciton $(|2\rangle)$ occurs nonradiatively, and the photoluminescence (PL) emitted from the $s$ shell is collected through the same objective lens and detected using a $0.75-\mathrm{m}$ monochromator and InGaAs array detector with a resolution of $30 \mu \mathrm{eV}$. For the QD under study, the $p$-shell and $s$-shell transitions are at 1.0688 and $0.9772 \mathrm{eV}$, respectively, determined using micro-PL and PL excitation techniques. For further details on the sample structure and experimental techniques, see Ref. [42].

\section{RESULTS}

\section{A. Ultrafast ARP}

We demonstrate ARP by measuring the PL intensity as a function of the pulse area $\Theta$ for increasing positive spectral chirp $\phi^{\prime \prime}$, which is applied to a transform-limited pulse using the pulse shaper [44]. Here the linearly chirped laser pulse is given by $E(t)=\frac{1}{2} E_{p}(t) \exp \left[-i\left(\omega_{l} t+\alpha t^{2}\right)\right]$, where $\omega_{l}$ is the center frequency of the laser pulse and the frequency is swept at rate $\alpha$, where $\alpha=2 \phi^{\prime \prime} /\left[\tau_{0}^{4} /(2 \ln (2))^{2}+\left(2 \phi^{\prime \prime}\right)^{2}\right]$, $\tau_{0}$ is the transform-limited pulse width, and $\tau_{p}=\tau_{0}[1+$ $\left.\left(4 \ln 2 \phi^{\prime \prime}\right)^{2} / \tau_{0}^{4}\right]^{1 / 2}$ is the width of the chirped pulse. The results of these experiments are shown in Fig. 1(c). A strongly damped Rabi oscillation is observed for zero pulse chirp, a signature of coherent manipulation of the exciton, as seen in previous work $[1-3,22,35]$. As the magnitude of the chirp is increased, the PL intensity for $\Theta>\pi$ increases, and a plateau in the emission emerges, corresponding to high inversion efficiency over a wider range of $\Theta$ as $\phi^{\prime \prime}$ is increased. For the largest value of chirp $\left(\phi^{\prime \prime}=0.133 \mathrm{ps}^{2}\right)$, the PL intensity is nearly independent of pulse area above the threshold for ARP. This trend was observed for both linearly and circularly polarized control pulses, indicating that biexciton dynamics do not play a significant role, consistent with previous studies of Rabi oscillations on the $p$-shell transition [35]. Figure 1(d) shows the PL intensity for a pulse area of $2 \pi$ as a function of the magnitude of the chirp (black circles), indicating a saturation behavior.

The insensitivity of the exciton PL intensity to changes in pulse area for a sufficiently large pulse chirp is a signature of adiabatic rapid passage and robust state inversion [7-12]. For ARP, coupling of a two-level system with a light field may be understood in terms of the instantaneous eigenstates of the system, the so-called dressed states $\left|\psi_{+}\right\rangle$and $\left|\psi_{-}\right\rangle$. In the adiabatic regime, the system remains in one of the dressed states while the admixture of the bare QD states $(|0\rangle$ and $|1\rangle)$ is evolved to invert the two-level system. The energy splitting of the states is given by $\sqrt{\Omega(t)^{2}+\Delta(t)^{2}}$, where $\Omega(t)=(\sqrt{2 \ln 2})\left(\Theta / \sqrt{\pi \tau_{0} \tau_{p}}\right) \exp \left[-2 \ln (2) t^{2} / \tau_{p}^{2}\right]$ is the instantaneous Rabi frequency and $\Delta(t)=2 \alpha t$ is the detuning of the laser field from the transition. For the positive chirp applied in the experiments in Fig. 1(c), the system evolves from the ground state to the exciton through the anticrossing in state $\left|\psi_{-}\right\rangle$, corresponding to traversal along the lowerenergy adiabatic branch (see inset in Fig. 2). Using the 


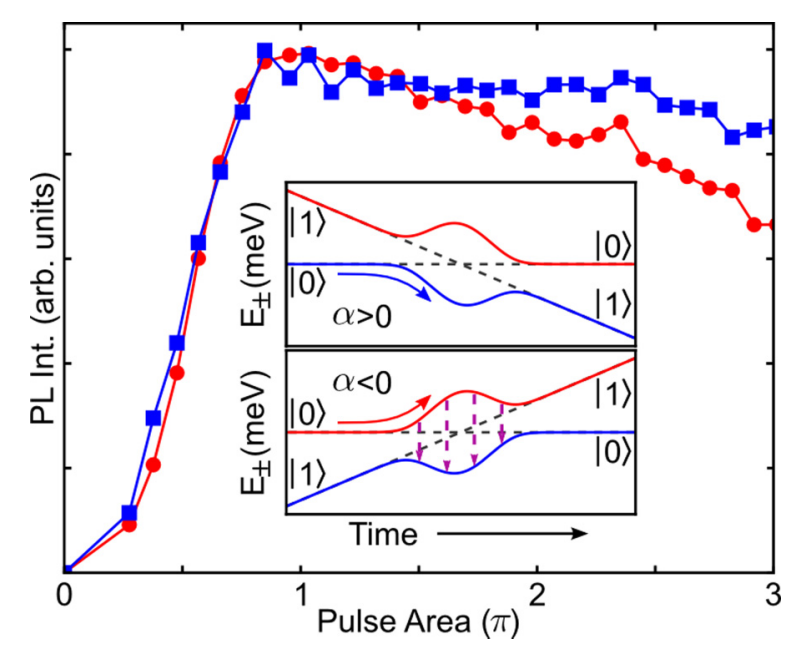

FIG. 2. (Color online) PL intensity as a function of pulse area for $\phi^{\prime \prime}=+0.133 \mathrm{ps}^{2}$ (blue squares) and $\phi^{\prime \prime}=-0.133 \mathrm{ps}^{2}$ (red circles). Inset: Illustration of the temporal evolution of the energies of the dressed states for positively chirped and negatively chirped pulses. The vertical dashed arrows represent diabatic transitions caused by phonon emission.

condition $\phi_{\min }^{\prime \prime} \approx \pi \tau_{0}^{2} /[2 \ln (2)]$ from Ref. [10], we estimate the minimum chirp required to achieve ARP to be $0.033 \mathrm{ps}^{2}$ in our experiments, corresponding to a pulse width of $\tau_{p}=$ $770 \mathrm{fs}$. This represents a reduction in the gate time for exciton inversion using ARP by a factor of $\gtrsim 20$ relative to previous demonstrations $[13,14]$. The use of broadband pulses in our experiments has also resulted in a lower threshold spectral chirp $\left(0.033 \mathrm{ps}^{2}\right.$ here, compared to $\sim 10 \mathrm{ps}^{2}$ in Refs. [13,14]). Furthermore, the requisite chirp is much easier to implement with standard commercially available frequency-domain pulse shapers when femtosecond pulses are used.

The achievement of ARP using subpicosecond pulses demonstrated here is greatly beneficial for quantum computing applications because it permits more gate operations within the decoherence time $[11,17-20,45]$. While this benefit is universal, the protective effect of short pulse control is particularly evident for our experiments, in which efficient ARP is achieved for the first time on the $p$-shell transition in a semiconductor QD despite a short-energy relaxation time to the $s$ shell [46,47]. Our demonstration of a subpicosecond gate also paves the way for strategies for decoherence protection based on dynamical decoupling [36-39], for which faster gates lead to greater protection. The ease and flexibility of pulse shaping in the femtosecond regime also permit more accurate dispersion compensation [43] as well as the ability to pursue arbitrary (nonlinear) phase control that can be used to optimize gate performance (e.g., fidelity, speed) [20,27,40-42] and may enable parallel processing in solid-state qubit systems [42]. The use of broadband pulses is aided by the strong confinement in the QDs studied here, which are characterized by a large energy separation between the $s$-shell and $p$-shell transitions and between the $p$-shell and the wetting-layer transitions (90 and $310 \mathrm{meV}$, respectively). The latter separation is expected to strongly diminish sources of decoherence associated with coupling to unwanted excitations in the wetting layer $[34,35]$.

\section{B. Role of phonons}

In order to investigate the role of phonons in our experiments, measurements were made of the dependence of the PL intensity on the sign of the pulse chirp (i.e., the sign of $\alpha$ ). The results of these experiments (for which $\left|\phi^{\prime \prime}\right|=0.133 \mathrm{ps}^{2}$, well above the threshold for ARP) are shown in Fig. 2. The PL intensity from the exciton transition for negative pulse chirp (red circles) is lower than that for positive pulse chirp (blue squares) by an amount that increases with increasing pulse area. This chirp sign dependence is observed for both linearly and circularly polarized excitation, indicating a negligible role of biexcitons in this effect. For $\alpha<0$, the system evolves from the ground state to the exciton through the anticrossing via the higher-energy adiabatic branch. In this case, diabatic transitions can occur in the vicinity of the anticrossing due to the emission of phonons [22-24,27,31]. In contrast, for $\alpha>0$, state evolution proceeds via the lower-energy adiabatic branch. Phonon-mediated transitions to the upper branch would occur through phonon absorption in this case, which is suppressed at $10 \mathrm{~K}[10,25,26,48]$. Phonon-mediated transitions between the adiabatic branches are a form of excitation-induced dephasing (EID) since the decoherence rate is dictated by the characteristics of the optical pulse. The results in Fig. 2 therefore indicate that coupling to phonons is the primary mechanism limiting the efficiency of exciton inversion via ARP and that the proper choice of the sign of the pulse chirp can diminish such effects at low temperatures. The protective effect of the energy gap between the higher- and lower-energy adiabatic branches should exist for temperatures $T$ for which $k_{B} T \lesssim \hbar \Lambda(t)$, where $\Lambda(t)=\sqrt{\Omega(t)^{2}+\Delta(t)^{2}}$ is the instantaneous value of the effective Rabi frequency. A reduced exciton inversion for negative pulse chirp was observed within the range of accessible temperatures in our experiments $(\leqslant 25 \mathrm{~K}$, limited by the noise floor of the InGaAs detector and the micro-PL technique used here for quantum state readout). This is consistent with the large Rabi energies in our experiments, as $\hbar \Lambda_{p}$ is approximately $7 \mathrm{meV}$, representing an enhancement of more than an order of magnitude compared to previous work [13,14]. The strongdriving conditions achieved in our experiments through the use of subpicosecond control pulses is therefore beneficial for the potential operation of devices that exploit ARP at elevated temperatures.

The identification of the physical process limiting the quality of quantum control is possible here because the sign of the pulse chirp in ARP provides a means to isolate phononrelated decoherence, as contributions to EID tied to wettinglayer excitations exhibit no such chirp sign dependence. The general conclusion of the dominance of phonon coupling also translates to other control processes such as Rabi rotations, for which the system is in a superposition of the higher- and lower-energy dressed states during optical control. In the case of Rabi oscillations, distinguishing between the wetting-layer and phonon contributions to EID represents a more formidable challenge $[22,23,33-35]$ as both mechanisms lead to similar trends in the dependence of the dephasing rate on pulse bandwidth and $\Theta$ (for small $\Theta$ ). Recent measurements of Rabi oscillation damping over a broad range of experimental conditions $[22,23]$ point to the dominance of phonon-mediated 
EID, in agreement with the findings we report here. For ARP, the sensitivity of exciton inversion efficiency on the sign of the pulse chirp demonstrated here provides a clear isolation of phonon-mediated EID. A weak decay at the highest pulse areas for $\alpha>0$ in the results in Fig. 2 may indicate a weak contribution from the wetting-layer mechanism, which increases in strength with increasing $\Theta$ [34,35], although the broad range of the pulse area with a relatively constant PL intensity in Fig. 2 is promising for applications utilizing ARP for exciton inversion.

While the above results clearly indicate the dominant role of phonons in dephasing for optical control on single semiconductor QDs, the relatively unexplored regime of strong (and rapid) driving of the coupled quantum-dot-phonon system considered here may also aid in the development of strategies for decoherence mitigation. In particular, the influence of phonon-mediated dephasing is dictated by the instantaneous value of $\Lambda(t)$ for times during the state evolution in the vicinity of the anticrossing, together with the frequency dependence of the phonon response function $K(\omega)[10,22,23,25,26,31]$. The exciton-phonon coupling is strongest when $K(\omega=\Lambda)$ is large as in this case the phonon modes can respond resonantly to laser-induced driving of the exciton at the effective Rabi frequency, resulting in phonon-mediated transitions between the adiabatic branches. The finite size of the exciton wave function within the quantum dot leads to a nonmonotonic frequency dependence of $K(\omega)$ that translates into a nonmonotonic dependence of the dephasing rate on the effective Rabi frequency: For small $\Lambda$, the phonon coupling increases with $\Lambda$, while for sufficiently large $\Lambda$, the phonon coupling may be reduced considerably due to the finite response time of the phonon bath. The latter result favors the execution of optical control on very short time scales since the peak value of $\Omega$ (and therefore of $\Lambda$ ) is given by $\Omega_{p}=(\sqrt{2 \ln 2})\left(\Theta / \sqrt{\pi \tau_{0} \tau_{p}}\right)$, which is strongly enhanced for a given pulse area in the experiments reported here due to the small values of $\tau_{p}$ and $\tau_{0}$.

In order to gain insight into the transition between the regime of Rabi frequencies considered in past demonstrations of ARP $[13,14]$ and the experiments in Fig. 2, numerical simulations of the quantum state evolution were carried out using a density-matrix approach [41] in which deformation coupling to acoustic phonons was included, taking into account pure dephasing via the real part of the complex phonon response function, following the model presented in Ref. [24]. The dependence of the exciton inversion efficiency on the sign of the pulse chirp was calculated for four different values of $\tau_{0}$, taking $\phi^{\prime \prime}=4 \pi \tau_{0}^{2} /(2 \ln 2)$, i.e., four times the threshold chirp for ARP discussed above. For $\tau_{0}=120 \mathrm{fs}$, this corresponds to $\phi^{\prime \prime}=0.131 \mathrm{ps}^{2}$, reflecting the experimental conditions for the results in Fig. 2. The results of these calculations are shown in Figs. 3(a)-3(d). The general trends are in line with the expected dependence on $\Omega_{p}$ discussed above. For the largest value of $\tau_{0}$ [825 fs; Fig. 3(a)], phonon-mediated dephasing results in a difference between the exciton occupation for positive and negative chirp that increases with increasing pulse area, similar to the trend observed experimentally (Fig. 2). As $\tau_{0}$ decreases, the coupling to phonons becomes most prominent for lower values of pulse area until $\tau_{0}=120 \mathrm{fs}$, where the model indicates
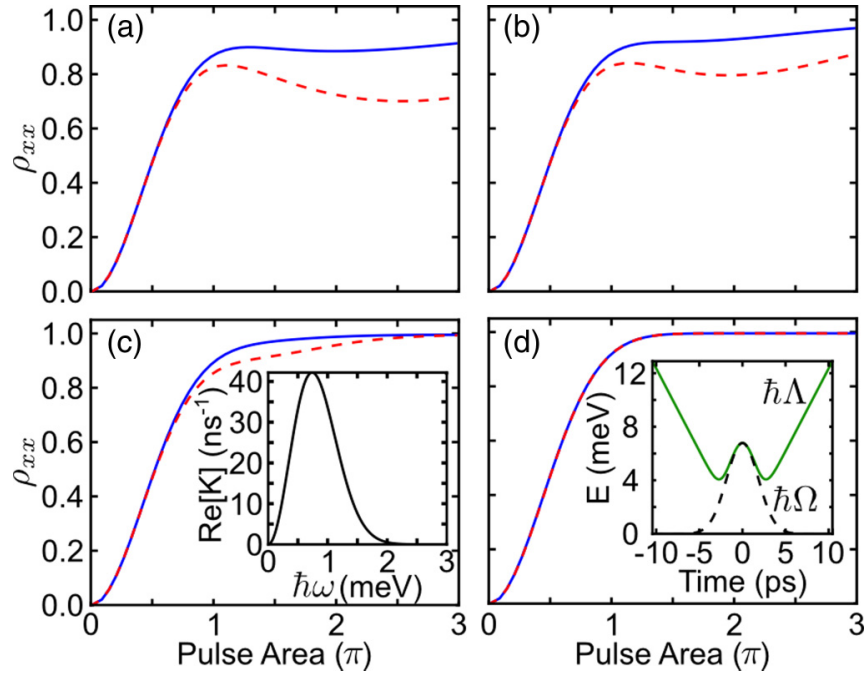

FIG. 3. (Color online) Calculated occupation of the exciton for positively chirped (solid blue curve) and negatively chirped (dashed red curve) pulses as a function of pulse area for increasing pulse bandwidth (i.e., decreasing $\tau_{0}$ ). The chirp applied to each pulse (dictating the chirped pulse duration $\tau_{p}$ ) is four times the threshold value, as described in the main text. (a) $\tau_{0}=825 \mathrm{fs}$. (b) $\tau_{0}=650 \mathrm{fs}$. (c) $\tau_{0}=410$ fs. Inset: Spectral dependence of the real part of the phonon response function. (d) $\tau_{0}=120 \mathrm{fs}$. Inset: Temporal evolution of the Rabi energy $\hbar \Omega$ (dashed black curve) and the effective Rabi energy $\hbar \Lambda$ (solid green curve) for a chirp of $\phi^{\prime \prime}=0.133 \mathrm{ps}^{2}$. Values of material parameters governing the strength of the phonon coupling were taken from Ref. [10].

negligible coupling to phonons, in contrast to the experimental results.

The quantitative deviations between the experimental results and the model considered here (i.e., the pulse bandwidth at which good agreement is obtained) provide insight into coherent control in the strong-field regime. In particular, the persistence of phonon-mediated dephasing in our experiments despite the large instantaneous Rabi frequencies suggests that other physical processes not included in the model of exciton-phonon coupling considered here may play a role and must be considered in the design of control pulses to minimize exciton-phonon coupling. While coupling to LO phonons $[9,32,49]$ may contribute to the strong damping of the Rabi oscillation in the zero-chirp results of Fig. 1(c), for the pulse characteristics used in the experiments of Fig. 2, coupling to LO phonons may be neglected since the peak Rabi energy remains below $7 \mathrm{meV}$ [see inset in Fig. 3(d)]. While the addition of piezoelectric coupling would enhance the phonon-mediated dephasing somewhat [49], for the conditions of our experiments in which the Rabi energy exceeds $4 \mathrm{meV}$ during the control pulse it may also be necessary to include multiphonon emission processes, which are absent in the model due to the perturbative treatment of exciton-phonon coupling [24,29]. In addition, the rapid variation of the laser pulse parameters in our experiments may necessitate a more accurate treatment of non-Markovian (memory) effects in the dynamics of the phonon bath than in the model considered here $[25,27-30]$. Our findings suggest that simply increasing 
the Rabi frequency may not be enough for decoherence mitigation when considering quantum state control at elevated temperatures. Alternate techniques such as the so-called bangbang protocol of dynamic decoupling with engineered pulse trains may provide a more effective strategy [36-39], for which the short time scale of ARP demonstrated here would be an important asset.

\section{CONCLUSIONS}

In summary, we have demonstrated adiabatic rapid passage in an experimental regime by using control pulses that are more than an order of magnitude shorter than those used in previous ARP experiments. The combination of a subpicosecond control time with the robustness of ARP achieved in this work will support the application of QDs in quantum information science by enabling more quantum manipulations within the decoherence time. Our results demonstrate the dominant role played by coupling to phonons in optical control experiments in single QDs by isolating such effects from other sources of decoherence through a dependence of the exciton inversion efficiency on the sign of the control pulse chirp. The importance of phonon-mediated dephasing is confirmed despite the large instantaneous Rabi frequencies in our experiments, which explore the limit of strong (and rapid) driving of the coupled exciton-phonon system.

\section{ACKNOWLEDGMENTS}

The authors thank the Natural Sciences and Engineering Research Council of Canada and Lockheed Martin Corporation for financial support.
[1] T. H. Stievater, X. Li, D. G. Steel, D. Gammon, D. S. Katzer, D. Park, C. Piermarocchi, and L. J. Sham, Phys. Rev. Lett. 87, 133603 (2001).

[2] H. Kamada, H. Gotoh, J. Temmyo, T. Takagahara, and H. Ando, Phys. Rev. Lett. 87, 246401 (2001).

[3] H. Htoon, T. Takagahara, D. Kulik, O. Baklenov, A. L. Holmes, and C. K. Shih, Phys. Rev. Lett. 88, 087401 (2002).

[4] D. Press, K. De Greve, P. L. McMahon, T. D. Ladd, B. Friess, C. Schneider, M. Kamp, S. Hofling, A. Forchel, and Y. Yamamoto, Nat. Photon. 4, 367 (2010).

[5] D. Kim, S. G. Carter, A. Greilich, A. S. Bracker, and D. Gammon, Nat. Phys. 7, 223 (2011).

[6] K. De Greve, L. Yu, P. L. McMahon, J. S. Pelc, C. M. Natarajan, N. Y. Kim, E. Abe, S. Maier, C. Schneider, M. Kamp, S. Hofling, R. H. Hadfield, A. Forchel, M. M. Fejer, and Y. Yamamoto, Nature (London) 491, 421 (2012).

[7] N. V. Vitanov, T. Halfmann, B. W. Shore, and K. Bergmann, Annu. Rev. Phys. Chem. 52, 763 (2001).

[8] J. S. Melinger, S. R. Gandhi, A. Hariharan, D. Goswami, and W. S. Warren, J. Chem. Phys. 101, 6439 (1994).

[9] K. Schuh, F. Jahnke, and M. Lorke, Appl. Phys. Lett. 99, 011105 (2011).

[10] A. Debnath, C. Meier, B. Chatel, and T. Amand, Phys. Rev. B 86, 161304 (2012).

[11] E. M. Gauger, S. C. Benjamin, A. Nazir, and B. W. Lovett, Phys. Rev. B 77, 115322 (2008).

[12] E. R. Schmidgall, P. R. Eastham, and R. T. Phillips, Phys. Rev. B 81, 195306 (2010).

[13] Y. Wu, I. M. Piper, M. Ediger, P. Brereton, E. R. Schmidgall, P. R. Eastham, M. Hugues, M. Hopkinson, and R. T. Phillips, Phys. Rev. Lett. 106, 067401 (2011).

[14] C.-M. Simon, T. Belhadj, B. Chatel, T. Amand, P. Renucci, A. Lemaitre, O. Krebs, P. A. Dalgarno, R. J. Warburton, X. Marie, and B. Urbaszek, Phys. Rev. Lett. 106, 166801 (2011).

[15] P. Michler, A. Kiraz, C. Becher, W. V. Schoenfeld, P. M. Petroff, L. Zhang, E. Hu, and A. Imamoglu, Science 290, 2282 (2000).

[16] A. Dousse, J. Suffczynski, A. Beveratos, O. Krebs, A. Lemaitre, I. Sagnes, J. Bloch, P. Voisin, and P. Senellart, Nature (London) 466, 217 (2010).
[17] T. Calarco, A. Datta, P. Fedichev, E. Pazy, and P. Zoller, Phys. Rev. A 68, 012310 (2003).

[18] B. W. Lovett, A. Nazir, E. Pazy, S. D. Barrett, T. P. Spiller, and G. A. D. Briggs, Phys. Rev. B 72, 115324 (2005).

[19] C. Creatore, R. T. Brierley, R. T. Phillips, P. B. Littlewood, and P. R. Eastham, Phys. Rev. B 86, 155442 (2012).

[20] H. Y. Hui and R. B. Liu, Phys. Rev. B 78, 155315 (2008).

[21] P. R. Eastham and R. T. Phillips, Phys. Rev. B 79, 165303 (2009).

[22] A. J. Ramsay, T. M. Godden, S. J. Boyle, E. M. Gauger, A. Nazir, B. W. Lovett, A. M. Fox, and M. S. Skolnick, Phys. Rev. Lett. 105, 177402 (2010).

[23] A. J. Ramsay, A. V. Gopal, E. M. Gauger, A. Nazir, B. W. Lovett, A. M. Fox, and M. S. Skolnick, Phys. Rev. Lett. 104, 017402 (2010).

[24] A. J. Ramsay, T. M. Godden, S. J. Boyle, E. M. Gauger, A. Nazir, B. W. Lovett, A. V. Gopal, A. M. Fox, and M. S. Skolnick, J. Appl. Phys. 109, 102415 (2011).

[25] S. Lüker, K. Gawarecki, D. E. Reiter, A. Grodecka-Grad, V. M. Axt, P. Machnikowski, and T. Kuhn, Phys. Rev. B 85, 121302 (2012).

[26] D. E. Reiter, S. Lüker, K. Gawarecki, A. Grodecka-Grad, P. Machnikowski, V. M. Axt, and T. Kuhn, Acta Phys. Pol. A 122, 1065 (2012).

[27] P. R. Eastham, A. O. Spracklen, and J. Keeling, Phys. Rev. B 87, 195306 (2013).

[28] A. Vagov, M. D. Croitoru, V. M. Axt, T. Kuhn, and F. M. Peeters, Phys. Rev. Lett. 98, 227403 (2007).

[29] D. P. S. McCutcheon and A. Nazir, New J. Phys. 12, 113042 (2010).

[30] J. Förstner, C. Weber, J. Danckwerts, and A. Knorr, Phys. Rev. Lett. 91, 127401 (2003).

[31] P. Machnikowski and L. Jacak, Phys. Rev. B 69, 193302 (2004).

[32] V. Axt, M. Herbst, and T. Kuhn, Superlattices Microstruct. 26, 117 (1999).

[33] L. Monniello, C. Tonin, R. Hostein, A. Lemaitre, A. Martinez, V. Voliotis, and R. Grousson, Phys. Rev. Lett. 111, 026403 (2013). 
[34] A. Vasanelli, R. Ferreira, and G. Bastard, Phys. Rev. Lett. 89, 216804 (2002).

[35] Q. Q. Wang, A. Muller, P. Bianucci, E. Rossi, Q. K. Xue, T. Takagahara, C. Piermarocchi, A. H. MacDonald, and C. K. Shih, Phys. Rev. B 72, 035306 (2005).

[36] L. Viola and S. Lloyd, Phys. Rev. A 58, 2733 (1998).

[37] V. M. Axt, P. Machnikowski, and T. Kuhn, Phys. Rev. B 71, 155305 (2005).

[38] T. E. Hodgson, L. Viola, and I. D'Amico, Phys. Rev. B 78, 165311 (2008).

[39] P. Karbach, S. Pasini, and G. S. Uhrig, Phys. Rev. A 78, 022315 (2008).

[40] A. Gamouras, R. Mathew, and K. C. Hall, J. Appl. Phys. 112, 014313 (2012).

[41] R. Mathew, C. E. Pryor, M. E. Flatté, and K. C. Hall, Phys. Rev. B 84, 205322 (2011).

[42] A. Gamouras, R. Mathew, S. Freisem, D. G. Deppe, and K. C. Hall, Nano Lett. 13, 4666 (2013).
[43] V. V. Lozovoy, I. Pastirk, and M. Dantus, Opt. Lett. 29, 775 (2004).

[44] The average power required to reach a given pulse area is proportional to the laser pulse bandwidth, limiting the maximum pulse area that avoids sample heating effects.

[45] W. C. Campbell, J. Mizrahi, Q. Quraishi, C. Senko, D. Hayes, D. Hucul, D. N. Matsukevich, P. Maunz, and C. Monroe, Phys. Rev. Lett. 105, 090502 (2010).

[46] H. Htoon, D. Kulik, O. Baklenov, A. L. Holmes, T. Takagahara, and C. K. Shih, Phys. Rev. B 63, 241303 (2001).

[47] T. F. Boggess, L. Zhang, D. G. Deppe, D. L. Huffaker, and C. Cao, Appl. Phys. Lett. 78, 276 (2001).

[48] The suppression of phonon absorption follows from the large value of the minimum splitting between the dressed states during the control pulse [approximately $4 \mathrm{meV}$; see Fig. 3(d), inset] relative to the thermal energy available at $10 \mathrm{~K}$.

[49] B. Krummheuer, V. M. Axt, and T. Kuhn, Phys. Rev. B 65, 195313 (2002). 